\title{
Quantum-dot growth simulation on periodic stress of substrate
}

\author{
Chang Zhao, ${ }^{\text {a) }}$ Y. H. Chen, ${ }^{\text {b) }}$ C. X. Cui, B. Xu, J. Sun, W. Lei, L. K. Lu, and Z. G. Wang \\ Key Laboratory of Semiconductor Materials Science, Institute of Semiconductors, \\ Chinese Academy of Sciences, P. O. Box 912, Beijing 100083, People's Republic of China
}

(Received 4 March 2005; accepted 29 June 2005; published online 8 September 2005)

\begin{abstract}
InAs quantum dots (QDs) are grown on the cleaved edge of an $\operatorname{In}_{x} \mathrm{Ga}_{1-x} \mathrm{As} / \mathrm{GaAs}$ supperlattice experimentally and a good linear alignment of these QDs on the surface of an $\operatorname{In}_{x} \mathrm{Ga}_{1-x}$ As layer has been realized. The modulation effects of periodic strain on the substrate are investigated theoretically using a kinetic Monte Carlo method. Our results show that a good alignment of QDs can be achieved when the strain energy reaches $2 \%$ of the atomic binding energy. The simulation results are in excellent qualitative agreement with our experiments. (C) 2005 American Institute of Physics. [DOI: 10.1063/1.2006677]
\end{abstract}

\section{INTRODUCTION}

Great efforts have been devoted to fabricating semiconductor quantum dots with spatial and size ordering due to their novel physical properties and potential applications. ${ }^{1-3}$ The self-assembly in the Stranski-Krastanow growth mode can produce quantum dots (QDs) with high optical qualities and size ordering which are, however, randomly distributed on the wafer surface. ${ }^{2}$ In many cases, a better control of the size and positional distribution of the QDs would be desirable. This has been mainly pursued by the selective overgrowth of artificially patterned substrates ${ }^{4,5}$ which is, however, strongly limited by the spatial resolution of the lithography and/or etching steps and easily introduces additional size fluctuations and defects in the QDs.

To overcome these problems, Romanov et al. ${ }^{6}$ investigated the possibility of using a periodic strain field on the surface of the substrate as a tool to control the nucleation of QDs, for example, an array of dislocations on the substrate. Recently, Wang et al. ${ }^{7}$ utilized the naturally curved surface contours provided by the oval defects on a GaAs(100) surface; an alignment of quantum-dot chains was realized. Nötzel et $a .^{8}{ }^{8}$ developed self-organized anisotropic strain engineering of (In, Ga)As/GaAs quantum wire superlattice templates on GaAs(100) substrates, and ordered groups of QDs were created. In this article we demonstrated the formation of InAs QDs in a very good linear alignment in an experiment by utilizing a periodic strain field on the cleaved edge of an $\mathrm{In}_{x} \mathrm{Ga}_{1-x} \mathrm{As} / \mathrm{GaAs}$ superlattice (SL); then a detailed kinetic Monte Carlo simulation of the growth process on this patterned substrate was studied.

Computer simulation had been proven to be an important and useful method in materials science and crystal growth ${ }^{9-11}$ and often provides a detailed description of the atoms' growth kinetics during the growth process. The effect of the strain field, as an important factor, was included by using a simple model in many simulations. ${ }^{12-15}$ Gsell et al. ${ }^{16}$ created strained regions on a surface and determined that the binding

\footnotetext{
a)Electronic mail: czhao@semi.ac.cn

${ }^{b)}$ Electronic mail: yhchen@red.semi.ac.cn
}

energy of the atoms adsorbed on it was modified. Wu and Metiu ${ }^{17}$ showed that the strain modifies substantially the adsorption isotherm of CO on $\mathrm{Pd}(100)$. Furthermore, Mattsson and Metiu ${ }^{18,19}$ used Monte Carlo simulations to study the nucleation of adsorbate islands on a solid surface on which a periodic strain field had been imposed. Their results showed that, in spite of its very small effect on the diffusion constant of the atoms, the strain field orders the ensemble of islands. In the heteroepitaxy of strained semiconductor systems such as InAs on GaAs, it is important to take into account the strain field which is generated during the growth process. Meixner et al. ${ }^{20,21}$ calculated the strain field in these systems by the elasticity theory.

In the present paper a novel and simple model is used to explore the effects of periodic strain on the substrate to the epitaxy growth of QDs. Firstly, we will introduce our experiment in Sec. II and then focus on the kinetic Monte Carlo (KMC) simulation of the experiment in Sec. III. Section IV presents the simulated results of the strain field effects on the alignment of QDs. A summary of the results can be found in Sec. V.

\section{EXPERIMENTAL PROCEDURE}

The sample was grown in a Riber-32 molecular-beam epitaxy (MBE) machine in two steps. It was prepared as follows (Fig. 1). First, it was grown on a semi-insulating GaAs(100) substrate and the native oxide layer on the substrate's surface was thermally removed at $480{ }^{\circ} \mathrm{C}$ under an $\mathrm{As}_{2}$ pressure of approximately $6.4 \times 10^{-6}$ torr. After the deposition of a 300 -nm-thick GaAs buffer layer at $480{ }^{\circ} \mathrm{C}$, the sample was cooled down to $422^{\circ} \mathrm{C}$ for the InGaAs/GaAs SL growth. Three $\operatorname{In}_{x} \mathrm{Ga}_{1-x} \mathrm{As} / \mathrm{GaAs}$ superlattice (in the growth direction, SL1: ten periods of 20-nm $\mathrm{In}_{0.05} \mathrm{Ga}_{0.95} \mathrm{As} / 80-\mathrm{nm}$ GaAs; SL2: ten periods of 12-nm $\mathrm{In}_{0.1} \mathrm{Ga}_{0.9} \mathrm{As} / 80-\mathrm{nm} \mathrm{GaAs}$; and SL3: seven periods of 8-nm $\mathrm{In}_{0.15} \mathrm{Ga}_{0.85} \mathrm{As} / 80-\mathrm{nm}$ GaAs and three periods of $8-\mathrm{nm}$ $\mathrm{In}_{0.15} \mathrm{Ga}_{0.85} \mathrm{As} / 20$-nm GaAs) were grown. They were separated by a $420-\mathrm{nm}$ GaAs. The GaAs buffer layer, spacer layer, and cap layer were all grown at $480{ }^{\circ} \mathrm{C}$, and the growth rate was $1.94 \AA / \mathrm{s}$; the $\mathrm{SL}$ was grown at $422^{\circ} \mathrm{C}$, and 


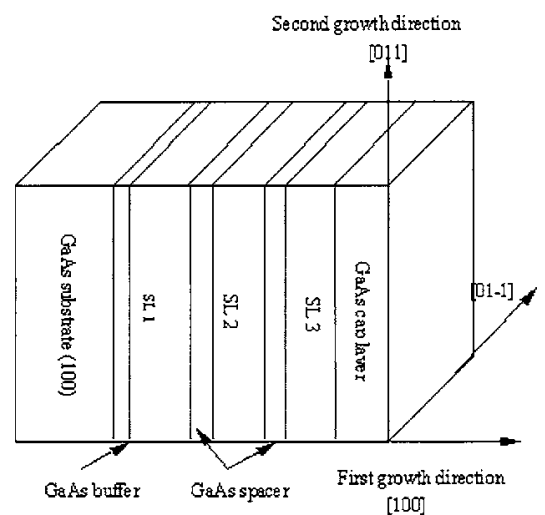

FIG. 1. Schematic illustration of the structure of the sample for cleaved edge overgrowth. In the first growth step the superlattice were grown on the GaAs(100) substrate. InAs were grown directly on the cleaved GaAs (011) surface in a subsequent MBE growth.

the growth rates of $\mathrm{In}_{0.05} \mathrm{Ga}_{0.95} \mathrm{As}, \mathrm{In}_{0.1} \mathrm{Ga}_{0.9} \mathrm{As}$, and $\mathrm{In}_{0.15} \mathrm{Ga}_{0.85}$ As were $1.61,1.70$, and $1.80 \AA / \mathrm{s}$, respectively.

After the first growth, the substrate was cleaved along the (011) crystal plane in the air, and remounted in the MBE. After being degassed for $20 \mathrm{~min}$ at $350{ }^{\circ} \mathrm{C}$, the sample was then loaded directly into the growth chamber of the MBE system. Desorbing the native oxide layer by annealing the substrate at $600{ }^{\circ} \mathrm{C}$ for $10 \mathrm{~min}$, the substrate temperature was reduced to $450{ }^{\circ} \mathrm{C}$ for the growth of a two-molecular layer (ML) InAs without growing the buffer layer. The growth rate of InAs was $0.06 \mathrm{ML} / \mathrm{s}$, and the $\mathrm{As}_{2}$ pressure was $5.3 \times 10^{-6}$ torr. The characterization of the surface morphology was performed by atomic force microscopy (AFM) in contact mode in air.

Figure 2 shows the AFM images of the sample. Positioncontrolled InAs QDs are realized and a distinct tendency of formation of InAs QD chains can be observed on the $x=0.1$ and $x=0.15$ sections of the $\operatorname{In}_{x} \mathrm{Ga}_{1-x}$ As/GaAs SL, with a poor ordering on the $x=0.05$ section [Figs. 2(a) and 2(b)]. The achievement of a good alignment of QDs in our experiment will be very useful for fundamental studies of quantum transport in low-dimensional structures and will have a potential application in nanoscale optoelectronic devices.

\section{KINETIC MONTE CARLO SIMULATIONS}

We simulate the ordering array of the InAs QDs' growth on the cleaved edge of an $\operatorname{In}_{x} \mathrm{Ga}_{1-x} \mathrm{As} / \mathrm{GaAs}$ SL by means of an event-based continuous kinetic Monte Carlo scheme. ${ }^{22-24}$ It is known that deposition, diffusion, desorption, and nucleation will all take place during the growth of the semiconductor material in the MBE system. In order to inhibit the atom desorption, some factors such as the low temperature and high pressure of $\mathrm{As}_{2}$ are adopted in the experiment. So in our simulation deposition and diffusion are considered as the main relevant processes. After the atoms' nucleation, they are still capable of diffusing unless their eight neighboring positions are all occupied by other atoms. In this simulation we only focus on a submonolayer heteroepitaxy growth. It is sufficient because we do not pay attention to the threedimensional (3D) morphology of an individual dot and

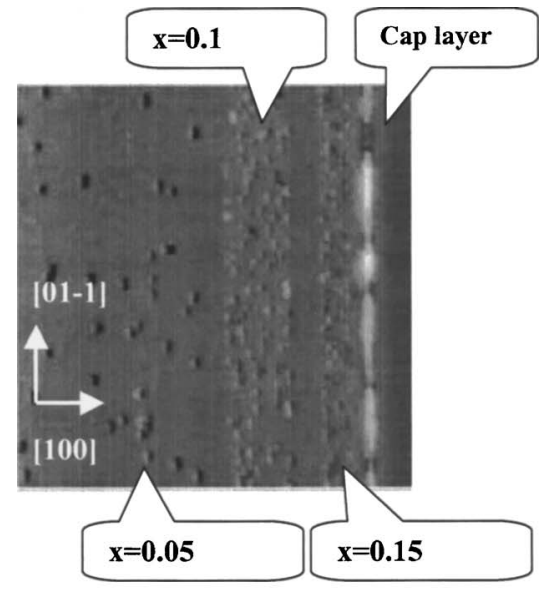

(a)

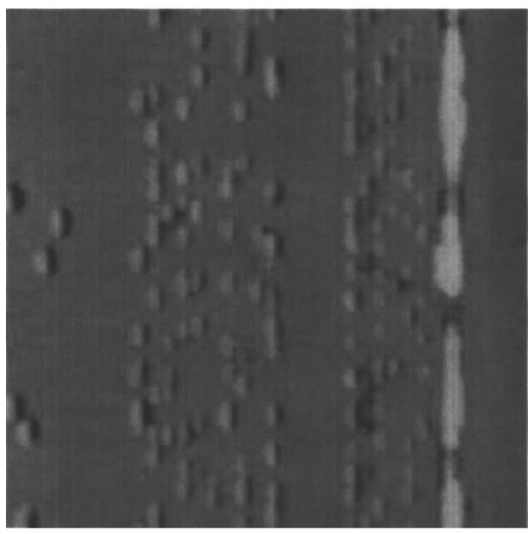

(b)

FIG. 2. AFM images of InAs deposited on the (011) cleaved edge with a growth temperature of $450{ }^{\circ} \mathrm{C}$. (a) The cleaved edge of a nanopattern, $5 \times 5 \mu \mathrm{m}^{2}$, with a growth rate of $f=0.06 \mathrm{ML} / \mathrm{s}$ and (b) an enlarged image of the $x=0.15$ and $x=0.1$ sections, $3 \times 3 \mu \mathrm{m}^{2}$.

mainly want to study whether an ordering quantum-dot array can form or not by the modulation of the periodic strain on the substrate's surface. In the simulation, after all the atoms have been deposited on the substrate, the system is allowed to equilibrate by a growth interruption.

Atom diffusion on a crystal surface is a thermally activated process: the hopping probability of an atom from one site to a neighboring one by overcoming a potential barrier $\Delta E$ is given by the Arrhenius law,

$$
\begin{aligned}
& P=v_{0} \exp \left(-\Delta E / k_{B} T\right), \\
& \Delta E=E_{s}+E_{N}+E_{\mathrm{AD}}-\Delta E_{\mathrm{STR}}, \\
& E_{N}=(n+\beta m) E_{n}-\varepsilon\left(n^{\prime}+\beta m^{\prime}\right) E_{n},
\end{aligned}
$$

where $E_{S}$ is the atomic binding energy to the surface and $E_{n}$ is that to a single nearest-neighbor atom and $n$ and $m$ are the numbers of the nearest- and next-nearest-neighbor atoms. $n^{\prime}$ and $m^{\prime}$ have the same meaning but correspond to the site to which the atom will hop. $E_{\mathrm{AD}}$ represents the energy barrier effect of anisotropy diffusion. It is necessary because in the experiment the cleaved surface is (011) oriented, so the hopping probabilities of atoms along different directions are unequal. $E_{\mathrm{STR}}$ is very important in the growth of semiconductor 


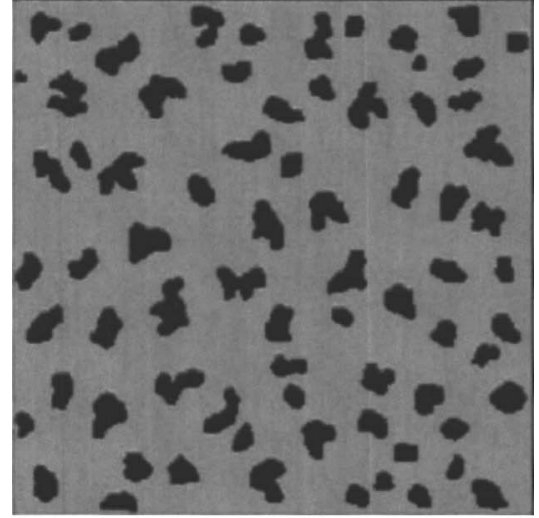

(a)

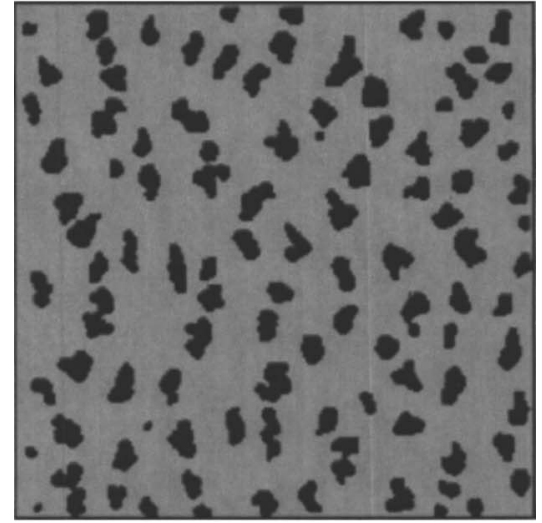

(b)

FIG. 3. Spatial distribution of atoms: the gray area represents the substrate and the black represents the QD. Simulation parameters are $T=723 \mathrm{~K}$, $f=0.06 \mathrm{ML} / \mathrm{s}$, growth time $=3 \mathrm{~s}$, and growth interruption $=50 \mathrm{~s}$ (a) without $E_{\mathrm{AD}}$ and $E_{\mathrm{STR}}$ and (b) with $E_{\mathrm{AD}}$ but without $E_{\mathrm{STR}}$.

materials and can modulate the nucleation sites of quantum dots, and this can be seen apparently from the AFM image of our sample (Fig. 2). In the experiment, it is easy to understand that if InAs is deposited on the $\operatorname{In}_{x} \mathrm{Ga}_{1-x}$ As layer, the $E_{\mathrm{STR}}$ on the deposited atom is smaller than that on the GaAs layer, and, of course, this further affects the atom's hopping probability. If InAs is deposited on the GaAs layer, the mismatch of the lattice is greatest and so is the $E_{\mathrm{STR}}$. In the simulation we let the $E_{\mathrm{STR}}$ be proportional to $1-x$, namely, be proportional to the degree of the lattice mismatch. So $E_{\mathrm{STR}}=E_{S}(1-x) c$, where $c$ is $7 \%$, i.e., the lattice mismatch between GaAs and InAs. When an atom crosses an island edge, a Schwöbel barrier of $0.1 \mathrm{eV}$ is also considered. Now all the important physical procedures which affect the diffusion of an atom are considered. The next task is to define a very little time interval corresponding to one Monte Carlo step during which there is only one atom that can diffuse. We compute the time interval by $\Delta t=1 / \Sigma p_{i}$. The most efficient search method developed to date is the binary tree process which is used in our simulation to find an atom to diffuse in every time interval. Some parameters used in the simulation are typical for a variety of semiconductor materials and equal to those of Ref. 20: $E_{S}=1.3 \mathrm{eV}, E_{n}=0.3 \mathrm{eV}, v_{0}$ $=10^{13} \mathrm{~s}^{-1}, \varepsilon=0.2, \beta=1 / \sqrt{2}, E_{\mathrm{AD}}=0.1 \mathrm{eV}$, and $k_{B}$ is Boltzmann's constant.

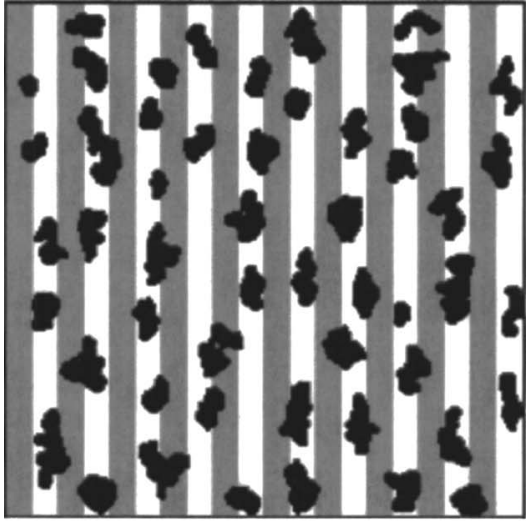

(a)

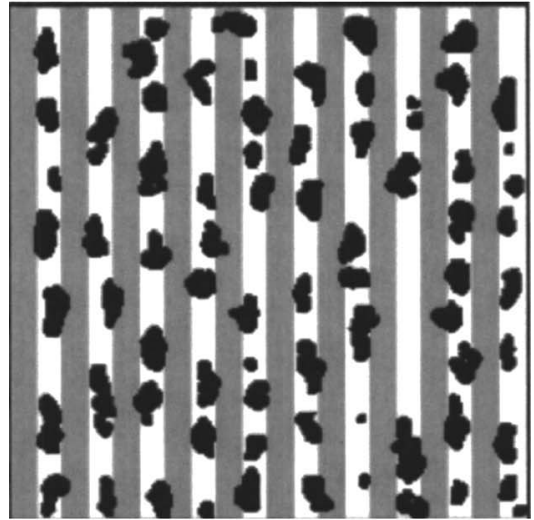

(b)

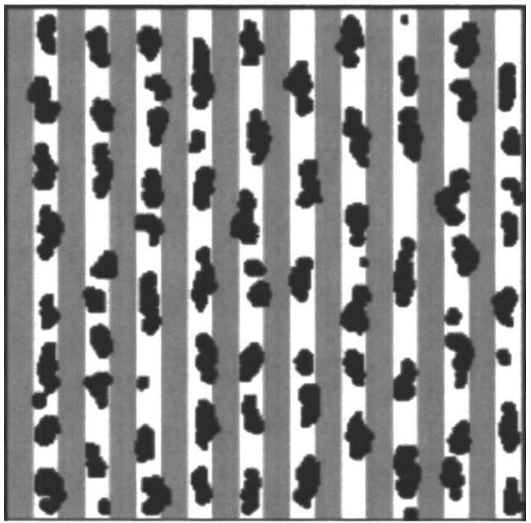

(c)

FIG. 4. The gray area represents the GaAs layers, and the white represents the $\operatorname{In}_{x} \mathrm{G}_{1-x}$ As layers: (a) $x=0.15$ in $\operatorname{In}_{x} \mathrm{G}_{1-x}$ As, (b) $x=0.3$, and (c) $x=0.5$. The other parameters are the same as those in Fig. 3.

\section{DISCUSSION ABOUT THE RESULTS OF SIMULATION}

All simulations are performed on a $200 \times 200$ grid (in units of the lattice constant). In order to investigate the effect of the $E_{\mathrm{AD}}$ and the periodic strain, we first simulate the growth without them, and some parameters such as the growth temperature and growth rate are the same as those in the experiment. Figure 3(a) shows the simulated result, where the two-dimensional QD distribution is random on the substrate's surface because everywhere it is the same with the deposited atom. When $E_{\mathrm{AD}}$ is considered the QDs be- 


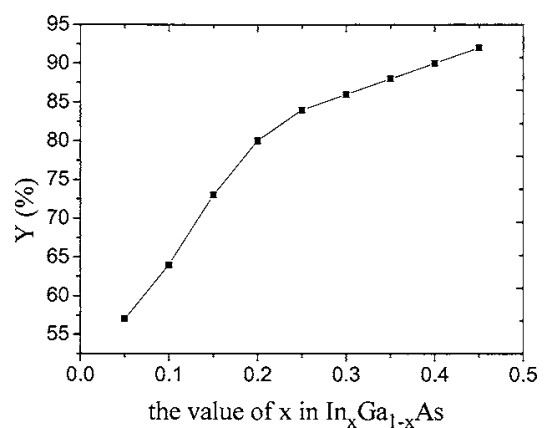

FIG. 5. $Y$ represents the ratio of atoms on the $\operatorname{In}_{x} \mathrm{Ga}_{1-x}$ As layers to the whole deposited atoms. The other parameters are the same as those in Fig. 3.

come elongated but without any alignment [Fig. 3(b)]. The fact that an independent elongated QD can be seen from our sample (Fig. 2) indicates that it is necessary to include $E_{\mathrm{AD}}$ in our simulation. Now we will add $E_{\mathrm{STR}}$ to the simulation, so all the factors discussed in Eq. (2) are considered. In order to compare the result with the experiment, the simulation area is divided into ten periods which is the same as each of the SL's periods in our sample. In Fig. 4, the gray area represents the GaAs layers and the white represents the $\mathrm{In}_{x} \mathrm{Ga}_{1-x} \mathrm{As}$ layers. We can find in Fig. 4(a) $(x=0.15)$ that the QDs appear aligned and are mainly on the white area, i.e., the $\mathrm{In}_{0.15} \mathrm{Ga}_{0.85}$ As layers. In Figs. 4(b) and 4(c), the values of $x$ in the $\mathrm{In}_{x} \mathrm{Ga}_{1-x} \mathrm{As} / \mathrm{GaAs} \mathrm{SL}$ are 0.3 and 0.5 , respectively, and a very good alignment of QDs can be seen. It indicates that under these conditions the nucleated position of the QDs can be modulated apparently by the periodic strain on the surface of the substrate. From the viewpoint of energy, all the simulation results can be explained by the fact that the $E_{\mathrm{STR}}$ on the GaAs layer is greater than that on the $\operatorname{In}_{x} \mathrm{Ga}_{1-x} \mathrm{As}$ layer, so the surface on the $\operatorname{In}_{x} \mathrm{Ga}_{1-x}$ As layer is the more comfortable place where the deposited atom can stay, and the deposited atoms that nucleate in this area will lead to a lower system energy. From the kinetic viewpoint, the atom on the surface of the GaAs layer can diffuse easily to the $\mathrm{In}_{x} \mathrm{Ga}_{1-x} \mathrm{As}$ layer but diffuse with difficulty inversely, so finally the QDs are mainly on the surface of the $\operatorname{In}_{x} \mathrm{Ga}_{1-x} \mathrm{As}$ layer. In order to illustrate quantitatively the selective growth of InAs QDs on the cleaved edge of the $\operatorname{In}_{x} \mathrm{Ga}_{1-x} \mathrm{As} / \mathrm{GaAs}$ $\mathrm{SL}$, we calculated the ratio of the atoms on the $\operatorname{In}_{x} \mathrm{Ga}_{1-x} \mathrm{As}$ layer to the whole deposited atoms. Ten simulations with different initial conditions are used to calculate the average ratio in order to reduce the noise and error. The ratio $Y$ as a function of $x$ is plotted in Fig. 5. When $x=0.05$ the ratio is $57 \%$ indicating that there is no apparent modulation effect of the periodic strain. The ratio reaches $64 \%$ when $x=0.1$, indicating an increase in the modulation effect of the periodic strain. When $x=0.15$ the ratio is $73 \%$, which implies that the modulation effect is apparent. After $x$ reaches 0.3 and 0.5 the ratio is $86 \%$ and $94 \%$, respectively, and now the periodic strain is strong enough to produce a very good alignment of QDs. At this time the $E_{\mathrm{STR}}$ is only $2.1 \%$ and $3.5 \%$ in relation to the $E_{S}$. This indicates that a little strain can seriously affect the growth of quantum dots, which is in accordance with the conclusion in Refs. 23 and 24 and has been realized in our experiment.

\section{SUMMARY AND CONCLUSIONS}

We fabricate semiconductor quantum dots which have very good spatial ordering, and through a KMC simulation we study the modulation effect of the periodic strain by using a novel model, in which the ratio between the strain energy and the local binding energy is proportional to the mismatch of the lattice. Both the experiment and the simulation produce a good alignment of QDs. The accordance between the experiment and the simulation indicates that the model in our simulation is reasonable. We find in the simulation that when the strain is greater than $2 \%$ of the local binding energy, it will have an important impact on the growth of QDs. This indicates that the utilization of periodic strain on the substrate's surface can be a promising method to produce a spatial ordering of quantum dots.

\section{ACKNOWLEDGMENTS}

The above work was supported by Special Funds for Major State Basic Research Project of China (No. G2000068303), National Natural Science Foundation of China (Nos. 60390071, 60390074, 90201007 and 90301007), and National High Technology Research and Development Program of China (No. 2002AA311070).

${ }^{1}$ D. Bimberg, M. Grundmann, and N. Ledentsov, Quantum Dot Heterostructures (Wiley, New York, 1999).

${ }^{2}$ W. Zhanguo, C. Yonghai, L. Fengqi, and X. Bo, J. Cryst. Growth 227228, 1132 (2001).

${ }^{3}$ I. Mukhametzhanov, R. Heitz, J. Zeng, P. Chen, and A. Madhukar, Appl. Phys. Lett. 73, 1841 (1998).

${ }^{4}$ S.-C. Lee, K. J. Malloy, and S. R. J. Brueck, J. Appl. Phys. 90, 4163 (2001).

${ }^{5}$ H. Lee, J. A. Johnson, J. S. Speck, and P. M. Petroff, J. Vac. Sci. Technol. B 18, 2193 (2000).

${ }^{6}$ A. E. Romanov, P. M. Petroff, and J. S. Speck, Appl. Phys. Lett. 74, 2280 (1999).

${ }^{7}$ Z. M. Wang, Y. I. Mazur, and G. J. Salamo, Appl. Phys. Lett. 84, 23 (2004).

${ }^{8}$ R. Nötzel, T. Mano, and J. H. Wolter, J. Vac. Sci. Technol. A 22, 1912 (2004).

${ }^{9}$ Z. Zhang, X. Chen, and M. G. Lagally, Phys. Rev. Lett. 73, 1829 (1994).

${ }^{10}$ B. G. Liu, J. Wu, E. G. Wang, and Z. Zhang, Phys. Rev. Lett. 83, 1195 (1999).

${ }^{11}$ T. R. Mattsson, G. Mills, and H. Metiu, J. Chem. Phys. 110, 12151 (1999).

${ }^{12}$ T. T. Ngo, P. M. Petroff, H. Sakaki, and J. L. Merz, Phys. Rev. B 53, 9618 (1996)

${ }^{13}$ C. S. Lee, B. Kahng, and A. L. Barabasi, Appl. Phys. Lett. 78, 984 (2001).

${ }^{14}$ P. M. Lam and S. Tan, Phys. Rev. B 64, 035321 (2001).

${ }^{15}$ E. Schöll and S. Bose, Solid-State Electron. 42, 1587 (1998).

${ }^{16}$ M. Gsell, P. Jakob, and D. Menzel, Science 280, 451 (1998).

${ }^{17}$ M. W. Wu and H. Metiu, J. Chem. Phys. 113, 1177 (2000).

${ }^{18}$ T. R. Mattsson and H. Metiu, Appl. Phys. Lett. 75, 926 (1999).

${ }^{19}$ T. R. Mattsson and H. Metiu, J. Chem. Phys. 113, 10323 (2000).

${ }^{20}$ M. Meixner, R. Kunert, and E. Scholl, Phys. Rev. B 67, 195301 (2003).

${ }^{21}$ M. Meixner, E. Scholl, V. A. Shchukin, and D. Bimberg, Phys. Rev. Lett. 87, 236101 (2001).

${ }^{22}$ S. V. Ghaisas and A. Madhukar, Appl. Phys. Lett. 53, 1599 (1988).

${ }^{23}$ K. E. Khor and S. Das Sarma, Phys. Rev. B 62, 16657 (2000).

${ }^{24}$ H. Shao, P. C. Weakliem, and H. Mitiu, Phys. Rev. B 53, 16041 (1996). 